\title{
A Migrated-mesenteric Lymphangioma: An Unusual Case of Intrabdominal Cystic Lesion in a New-born
}

\author{
(D) Fatma Ceren Sarıoğlu1, (1) Orkun Sarıoğlu2, (1) Deniz Öztekin³, (1) Emel Tekin4, (1) Aslı Irmak Birancı², \\ ๑ Özgür Öztekin² \\ 'Dokuz Eylül University Faculty of Medicine, Department of Radiology, İzmir, Turkey \\ ZUniversity of Health Sciences, İzmir Tepecik Training and Research Hospital, Clinic of Radiology, İzmir, Turkey \\ ${ }^{3}$ University of Health Sciences, İzmir Tepecik Training and Research Hospital, Clinic of Gynecology and Obstetrics, İzmir, Turkey \\ ${ }^{4}$ University of Health Sciences, İzmir Tepecik Training and Research Hospital, Clinic of Pathology, Izmir, Turkey
}

\begin{abstract}
Abdominal lymphangioma is a rare presentation of abdominal cystic lesions. They generally arise from small bowel mesentery. Migration of abdominal masses is an uncommon entity and usually occurs due to gossypiboma. There have been many reports of the radiologic appearance and unusual complications of mesenteric lymphangioma. However, to our knowledge, migration of mesenteric lymphangioma has not been reported in the literature. We present imaging findings of migrated-mesenteric lymphangioma from the intrauterine to postnatal period with histopathological correlation.
\end{abstract}

Keywords: Mesenteric lymphangioma, intraabdominal cyst, migration, CT, MRI

\section{Introduction}

Lymphangiomas are congenital benign malformations. They commonly occur in the head and neck region in children. Abdominal lymphangiomas are rare and defined as mesenteric lymphangioma, which is one of the subgroups of mesenteric cysts, in the case of origin from the mesentery (1). Although abdominal lymphangiomas are described most commonly in the small-bowel mesentery, they have been reported as less than $1 \%$ of all lymphangiomas (2).

There have been many reports of the radiologic appearance and unusual complications of mesenteric lymphangioma, however, to our knowledge, the migration of mesenteric lymphangioma has not been reported in the literature. We present imaging findings of migratedmesenteric lymphangioma from the intrauterine to postnatal period with histopathological correlation.

\section{Case Report}

A 35 -year-old primigravid pregnant woman at the $32^{\text {nd }}$ week of gestation presented to the perinatology department for a routine check-up. On ultrasound, the foetus had a $42 \times 35 \mathrm{~mm}$ cystic mass on the left side. The patient was referred to our clinic for determination of the origin of the cystic lesion by using foetal magnetic resonance imaging (MRI). The mass was located in mesentery and adjacent to the small bowel wall (Figure 1). There were no apparent 
fluid-fluid levels and fatty component, also the lesion did not originate from solid organs. The cystic lesion was determined as a mesenteric cyst. The patient was followedup and a baby girl weighing 2.950 grams was born at the $38^{\text {th }}$ week of gestation. Physical examination revealed a palpable, mobile mass on the left side of the abdomen. There was no physical abnormality, and routine laboratory tests were normal.

A contrast-enhanced computed tomography scan was performed on the postpartum second day and showed contrast enhancement of thin wall and septa. The left-sided intestinal bowel segment was surrounded by the cystic lesion (Figure 2). There were no air-fluid levels in the bowel segments. An operation was planned for one month later. Physical examination before surgery was confusing because the cystic lesion was not palpable on the left side. MRI was performed and it showed that the cystic lesion migrated to the right side while maintaining the same features and dimensions. The lesion was removed completely including the resection of the adjacent bowel involved by the cyst. Histopathological examination confirmed mesenteric lymphangioma. Informed consent was obtained from the parents.

\section{Discussion}

Foetal abdominal cystic lesions often arise from the genitourinary and gastrointestinal systems. Others are mesenteric cysts, meconium pseudocysts, and choledochal cysts. Location, relationships with abdominal organs and the internal structure of cysts are crucial for differential diagnosis. Mesenteric cysts are described as cysts which are located in the mesentery, omentum, and retroperitoneum

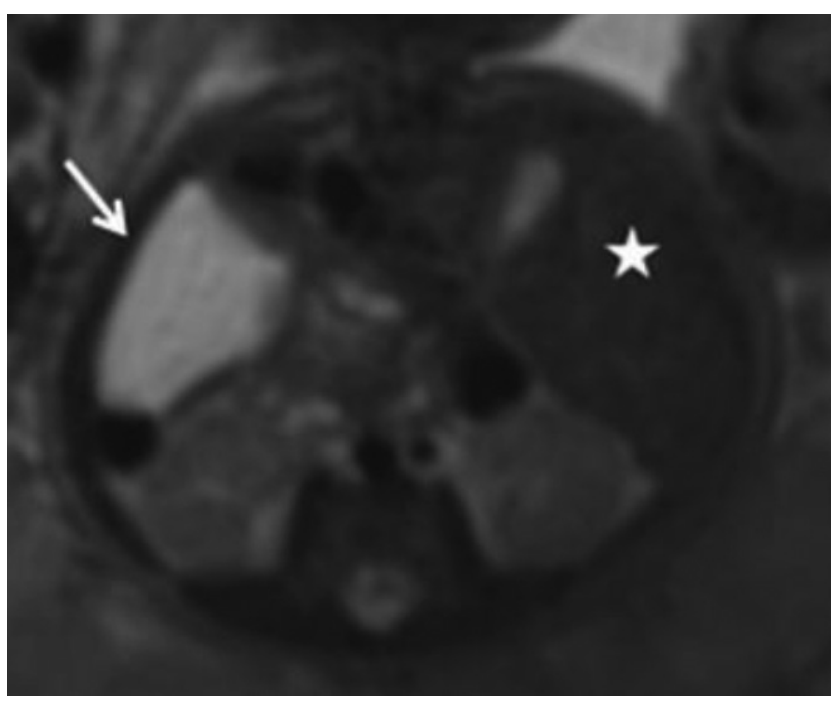

Figure 1. On axial T2-weighted foetal imaging shows hyperintense cystic lesion on the left side (arrow), note that liver is on the right side (star) and do not originate from solid organs $(1,3)$. They are divided into six groups which were previously described by de Perrot et al. (4) Mesenteric lymphangiomas are reported in children and young adults, however a small number of cases with mesenteric lymphangioma in new-borns have been reported in the literature.

The clinical presentation of a mesenteric lymphangioma is usually asymptomatic, but abdominal distension, abdominal pain, infection, haemorrhage, and ileus may occur (5). The most common finding during physical examination is a palpable abdominal mass. Also, mesenteric cysts can move in transverse directions but have limited movement vertically (3).

Imaging techniques play a major role in the determination of cysts and their relationships with abdominal organs. Lymphangiomas are usually seen as well defined, thinwalled, multiloculated cysts. Fluid-fluid levels might be seen depending on cyst contents (5).

Bhullar and Orfanou (6) reported on a mesenteric pseudocyst that would disappear and then reappear in different locations within the abdomen. They hypothesized
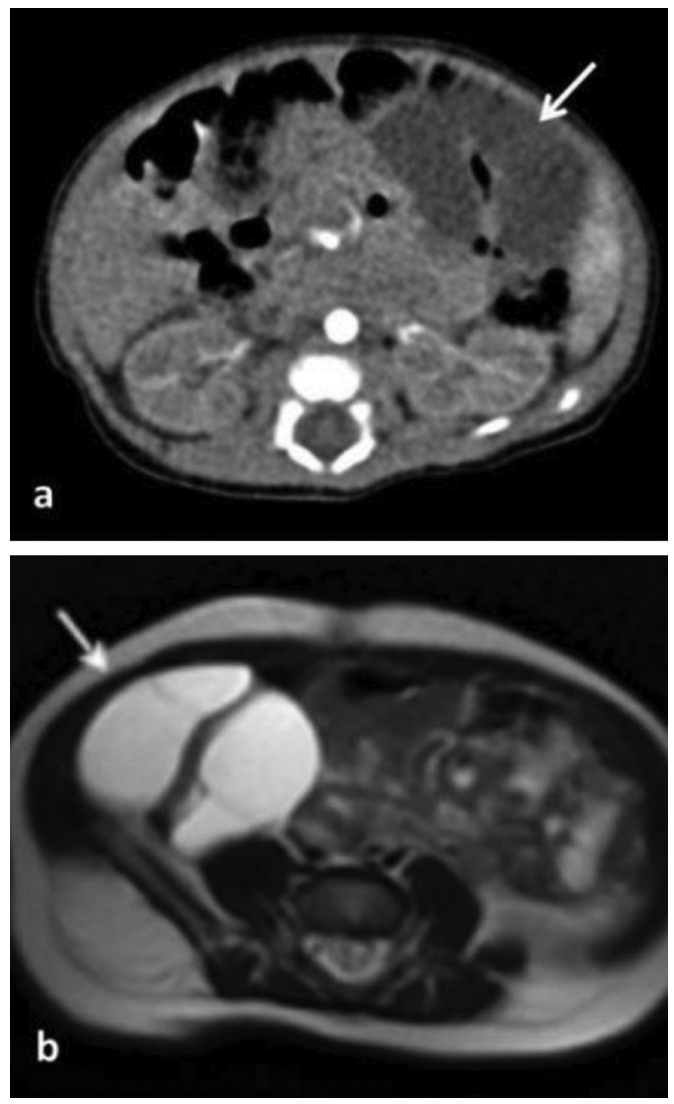

Figure 2. a) Axial computed tomography scan shows a well-defined, hypodense lesion on the left side (arrow) which surrounds the intestinal segment. b) Axial T2-weighted imaging shows migrated-cystic lesion from the left side to the right side (arrow). Note that internal septations and surrounded-intestinal segment are also seen 
that this situation is associated with the mobile jejunum. In our opinion, intestinal peristalsis might cause migration of the cystic lesion contralaterally. As a known fact, mesentery allows for movement of the organs during digestion.

Foetal abdominal cystic lesions generally have their origin in the genitourinary system, such as hydronephrosis, renal dysplasia, ovarian cyst, and urachal cyst. Additionally, atresia or duplication of gastrointestinal system may be the cause of abdominal cysts. Differential diagnosis might be a problem due to the small abdominal capacity of foetuses. However, there are some significant signs which help with the distinction between them. Furthermore, cystic lesions rarely arise from the adrenal glands, pancreas or spleen.

The definitive treatment for abdominal mesenteric lymphangioma is complete surgical excision. Because of the risk of recurrence and malignant transformation, a bowel resection is the recommended surgical technique based on the intimate relationship between the cyst and the intestine (7).

In conclusion, determination and accurate anatomic location of lymphangiomas are important in surgical planning. Migrated mesenteric lymphangioma is distinct from most of the previous cases. Migrating of the lesion contralaterally and emphasizing with imaging findings are the values of this case report.

\section{Ethics}

Informed Consent: It was taken from family.
Peer-review: External and internal peer-reviewed.

\section{Authorship Contributions}

Concept: F.C.S., Ö.Ö., Design: F.C.S., O.S., Data Collection or Processing: A.I.B., E.T., D.Ö., Analysis or Interpretation: F.C.S., Ö.Ö., Writing: F.C.S., O.S.

Conflict of Interest: No conflict of interest was declared by the authors.

Financial Disclosure: The authors declared that this study received no financial support.

\section{References}

1. Stoupis C, Ros PR, Abbitt PL, Burton SS, Gauger J. Bubbles in the belly: imaging of cystic mesenteric or omental masses. Radiographics 1994;14:729-37.

2. Suthiwartnarueput $W$, Kiatipunsodsai S, Kwankua A, Chaumrattanakul U. Lymphangioma of the small bowel mesentery: a case report and review of the literature. World I Gastroenterol 2012;18:6328-32.

3. Vanek VW, Phillips AK. Retroperitoneal, mesenteric and omental cysts. Arch Surg 1984;119:838-42.

4. de Perrot $M$, Bründler $M$, Tötsch $M$, Mentha $G$, Morel P. Mesenteric cysts. Toward less confusion? Dig Surg 2000;17:3238.

5. Levy AD, Cantisani V, Miettinen M. Abdominal lymphangiomas: imaging features with pathologic correlation. AJR Am J Roentgenol 2004;182:1485-91.

6. Bhullar JS, Orfanou P. The disappearing abdominal mass: mesenteric pseudocyst. JSLS 2014;18:319-22.

7. Kenney B, Smith B, Bensoussan AL. Laparoscopic excision of a cystic lymphangioma. J Laparoendoscop Surg 1996;6:99-101. 